Dirk Fox

\title{
Smart Meter
}

Der Stromverbrauch in Haushalten wird üblicherweise mit einem Ferraris-Zähler gemessen, einem nach dem italienischen Physiker Galileo Ferraris benannten elektromechanischen Messgerät. Der Stand des mechanischen Zählwerks wird jährlich von einem Mitarbeiter des Energieversorgers abgelesen.

Nachteil dieser Art der Verbrauchserfassung ist, dass die Berücksichtigung wechselnder Tarife mit vertretbarem Aufwand nur für größere Zeiteinheiten realisiert werden kann, z. B. durch eine Zwischenablesung des Kunden. Lange Ablesezeiträume erschweren außerdem die Verbrauchsvorhersage durch die Energieversorger; sie sind daher auf stark vereinfachte Standard-Lastprofile angewiesen, die lediglich grob zwischen Werktagen, Samstagen und Sonn- und Feiertagen sowie Jahreszeiten (Sommer, Winter, Übergangszeit) unterscheiden.

Dank elektronischer Energiezähler, auch als "Intelligente Stromzähler" oder "Smart Meter" bezeichnet, sollen diese Nachteile bald der Vergangenheit angehören. Smart Meter sollen die Steuerung des Verbraucherverhaltens durch zeitabhängige Tarife erlauben und die Genauigkeit der Verbrauchsprognosen verbessern.

\section{Funktionsumfang}

Smart Meter übermitteln entweder die Verbrauchswerte in einstellbaren Intervallen automatisch an den Energieversorger oder erlauben eine Fernabfrage. Verbreitet ist eine Übermittlung der Zählerstände in 15-Minuten-Intervallen. Die Übermittlung erfolgt unter Verwendung standardisierter Datenübertragungsverfahren über Funknetze (z. B. GSM, GPRS) oder leitungsgebunden (z. B. DSL).

Meist sind die Systeme auch in der Lage, die Messwerte in kleineren Zeiteinheiten (Sekunden) zu erfassen. Einige Geräte bieten zusätzlich die Möglichkeit, die Verbrauchsdaten über eine Erhebungsperiode zu speichern und mittels PC auszuwerten. Über einen Rückkanal werden z. T. auch der aktuelle Tarif oder die über eine Photovoltaik-Anlage eingespeiste Energiemenge angezeigt.
Mit zukünftigen Geräteversionen soll auch die automatische Steuerung einzelner Verbraucher im Haushalt möglich sein, sowie die Nutzung der Zähler als „Gateway“ für die Übermittlung anderer Verbrauchsdaten des Haushalts (Heizung, Wasser, Gas).

Vorteile

Smart Meter erlauben dem Energieversorger monatliche Rechnungsstellung nach dem tatsächlichen Verbrauch. Von der damit verbundenen Transparenz erhofft sich der Gesetzgeber einen bewussteren Umgang der Verbraucher mit Energie.

Umgekehrt gewinnt der Energieversorger wertvolle Informationen über das Verbrauchsverhalten, die ihm genauere Prognosen erlauben, wie z. B. den Einfluss von Großereignissen (Olympiade, Fußballweltmeisterschaft) auf den Energieverbrauch, und so eine Präzisierung der Standard-Lastprofile ermöglichen.

Durch das Angebot günstiger Tarife werden Energieversorger zukünftig versuchen, die Nutzung energieintensiver Geräte in Nebenzeiten zu verschieben. Damit ließe sich die Bereitstellung zusätzlicher Energie in Spitzenlastzeiten reduzieren.

Gefahren

Detaillierte Verbrauchsprofile werden nicht nur bei Strafverfolgungsbehörden Begehrlichkeiten wecken. So lassen sich aus den Lastprofilen Besitzer veralteter Waschund Spülmaschinen sowie Strom fressender Kühlgeräte herausfiltern - ideale Adressaten für das Angebot von Neugeräten. Wer gerne ausgiebig duscht könnte an einer neuen Brause interessiert sein, intensive Fernseh-Nutzer an einem neuen Bildschirm und Nutzer von Heizspiralen an Windel-Sonderangeboten. Wer oft mitten in der Nacht aufwacht benötigt vielleicht ein Schlafmittel, und wer häufig wäscht einen Trockner.

\section{Gestaltung}

Zentrale Bedeutung für die datenschutzrechtliche Bewertung von Smart Metern hat die konkrete Ausgestaltung des Leistungsumfangs.
So genügt es für eine von wechselnden Tarifen abhängige monatliche Rechnungstellung, die Verbrauchsberechnung im Zähler vorzunehmen und lediglich den Rechnungsbetrag monatlich an den Energieversorger zu übermitteln.

Umgekehrt erfordert eine gute Verbrauchsprognose kurze Messintervalle; die Messwerte können jedoch über eine Vielzahl von Haushalten aggregiert sein.

Der Endverbraucher hingegen möchte zur Verbrauchskontrolle die Werte seines Zählers mit möglichst hoher zeitlicher Auflösung selbst auswerten - ohne dass diese zuvor an den Energieversorger übermittelt werden.

Werden die Verbrauchsdaten via Internet an den Energieversorger übertragen, muss diese Verbindung durch Verschlüsselung vor unberechtigtem Zugriff geschützt werden.

Falls der Intelligente Stromzähler eine Fernabfrage erlaubt oder, wie bei einigen Anbietern, über einen eigenen Webserver verfügt, bei dem der Verbraucher seine Verbrauchsdaten online abfragen kann, muss dieser so konfiguriert und gehärtet sein, dass ein unberechtigter Datenzugriff ausgeschlossen ist. $\mathrm{Zu}$ erwarten ist, dass bei den Geräten regelmäßig Software-Updates aufgespielt werden müssen - ein weiterer möglicher Ansatzpunkt für Angreifer.

Werden Zähler ausgetauscht oder repariert, muss zudem für eine Datenschutz konforme Löschung der darin gespeicherten Verbrauchsdaten gesorgt werden.

\section{Zukünftige Entwicklung}

Es ist zu erwarten, dass die heute verfügbaren Smart Meter noch nicht das Ende der Entwicklung darstellen. Wirksamer noch als die Beeinflussung des Verbraucherverhaltens über günstige Tarife wäre eine direkte Steuerung von Geräten mit hohem Energiebedarf. Würde diese Steuerung durch den Energieversorger erfolgen, müsste dieser zahlreiche weitere Daten kennen: die Leistungskennzahlen und Lastprofile der zu steuernden Geräte, Ausschlusszeiten und Nutzungspräferenzen. Lastspitzen ließen sich so erheblich abmildern - die Menge der Informationen über die jeweiligen Haushalte und deren Mitglieder würden zugleich jedoch erheblich zunehmen. 